\title{
Conflicto cognitivo en la metamotivación para el desarrollo de capacidades en estudiantes universitarios
}

\author{
Cognitive conflict in metamotivation for capacity development in university students
}

Conflito cognitivo em metamotivação para o desenvolvimento de capacidades em estudantes universitários

\section{Teófilo Félix Valentín Melgarejo \\ tevame39@gmail.com \\ https://orcid.org/0000-0002-4063-5516}

\author{
José Rovino Alvarez López \\ joal14@gmail.com
}

https://orcid.org/0000-0002-0019-3872

\author{
Oscar Cirilo Rivera Trujillo \\ ocriverat@gmail.com \\ https://orcid.org/0000-0002-9692-0041 \\ Rosa Luz Gómez Segura \\ ross.luz.g.s@gmail.com \\ https://orcid.org/0000-0002-9617-4974
}

\section{Gastón Jeremías Oscátegui Nájera \\ gaston1567@gmail.com \\ https://orcid.org/0000-0003-3228-5365}

Universidad Nacional Daniel Alcides Carrión, Cerro de Pasco-Perú

\section{ABSTRACT}

La formación universitaria exige el uso de estrategias para el desarrollo del aprendizaje, sin embargo; se desconoce sobre las estrategias del conflicto cognitivo en la metamotivación para el desarrollo de capacidades. De ahí, se tuvo como objetivo relacionar el conflicto cognitivo con la metamotivación para determinar el impacto en el desarrollo de capacidades, mediante las situaciones problemáticas, nuevos saberes y necesidades de aprendizaje en los estudiantes de la Universidad Nacional Daniel Alcides Carrión. Al respecto, 32 estudiantes, conformada por 18 del grupo experimental y 14 del grupo de control, respondieron un cuestionario de 12 ítems para determinar la relación entre las variables de estudio y una prueba de 20 preguntas para conocer el desarrollo de las capacidades, que fue validada previamente. Como resultado final se obtuvo $-4,171$ con 30 grados de libertad, por lo que; el conflicto cognitivo tiene impacto positivo en la metamotivación determinando el desarrollo de capacidades profesionales.

Palabras clave: Aprendizaje; Capacidades; Conflicto cognitivo; Metamotivación; Proceso pedagógico

\section{RESUMEN}

University training requires the use of strategies for the development of learning, however; the strategies of cognitive conflict in metamotivation for capacity development are unknown. Hence, the objective was to relate cognitive conflict with metamotivation to determine the impact on the development of capacities, through problematic situations, new knowledge and learning needs in students of the Daniel Alcides Carrión National University. In this regard, 32 students, made up of 18 from the experimental group and 14 from the control group, answered a 12-item questionnaire to determine the relationship between the study variables and a 20-question test to know the development of capacities, which was previously validated. As a final result, $-4,171$ was obtained with 30 degrees of freedom, therefore; cognitive conflict has a positive impact on metamotivation, determining the development of professional skills.

Key words: Learning; Abilities; Cognitive conflict; Metamotivation; Pedagogical process

\section{RESUMO}

A formação universitária requer ou utiliza estratégias para o desenvolvimento de estágios, entretanto; as estratégias de conflito cognitivo em metamotivação para o desenvolvimento de capacidades até então desconhecidas. Assim, o objetivo foi relacionar o conflito cognitivo com a metamotivação para determinar o impacto no desenvolvimento de capacidades, através de situações problemáticas, novos conhecimentos e necessidades de aprendizagem em alunos da Universidade Nacional Daniel Alcides Carrión. Nesse sentido, 32 alunos, sendo 18 do grupo experimental e 14 do grupo controle, responderam a um questionário de 12 itens para determinar a relação entre as variáveis de estudo e a um teste de 20 questões para conhecer o desenvolvimento de capacidades, que foi previamente validado. Como resultado final, foi obtido $-4,171$ com 30 graus de liberdade, portanto; o conflito cognitivo tem impacto positivo na metamotivação, determinando o desenvolvimento de habilidades profissionais.

Palavras-chave: Aprendizagem; Habilidades; Conflito cognitivo; Metamotivação; Processo pedagógico 


\section{INTRODUCCIÓN}

Los grandes avances científicos y tecnológicos exigen mejorar las estrategias metodológicas en el sistema educativo y en el proceso de la enseñanza y aprendizaje, de manera que se pueda formar personas competitivas y capaces de auto realizarse, para enfrentar y solucionar problemas de su vida diaria y de su contexto; teniendo en cuenta la autónoma, práctica de valores éticos y reflexivos, responsable de sus ideas y respetando su modo de pensar de los demás; que le permita a la persona desenvolverse en contextos diferentes y según su diversidad, haciendo uso de estrategias para interactuar que le permita desarrollar sus capacidades cognitivas, obligando al sistema educativo establecer lineamientos que perduren en el tiempo y que se consideren significativos a fin de que las personas desarrollen hábitos que les permitan generar un aprendizaje significativo y duradero. Al respecto, uno de los fines de la educación peruana, considera formar personas capaces de lograr su realización ética, intelectual, artística, cultural, afectiva, física, espiritual y religiosa, promoviendo la formación y consolidación de su identidad para el ejercicio de su ciudadanía en armonía con su entorno, así como el desarrollo de sus capacidades, habilidades para vincularse con el mundo del trabajo y afrontar los incesantes cambios en la sociedad y el conocimiento (Ley No 28044, 2003).

Con la intención de lograr este fin, las últimas décadas se han caracterizado por la aplicación de mayores cambios en el sistema educativo, pero hasta la fecha se continúa con una gran cantidad de falencias en el proceso de enseñanza y aprendizaje y en el uso de estrategias pertinentes para el desarrollo de capacidades en la formación de docentes en la educación superior universitaria, con respecto al conflicto cognitivo del proceso pedagógico, siendo este un elemento primordial para promover y motivar los aprendizajes de manera significativa. El modelo del aprendizaje se ha difundido mucho más allá del universo de los educadores y ha penetrado en todos los poros de la vida económica y social, admitiendo cada vez más la necesidad de reforzar la dimensión educativa de cualquier organización, sea o no de carácter comercial (Organización de las Naciones Unidas para la Educación, la Ciencia y la Cultura, 2005).

Asimismo, la educación debe priorizar el reconocimiento de la persona como eje central del proceso educativo, donde la calidad asegure la eficiencia en los procesos y eficacia en los logros y las mejores condiciones de una educación para la identidad, la ciudadanía, el trabajo, en un marco de formación permanente (Ministerio de Educación, 2005).

Para ello es necesario fomentar un aprendizaje autónomo con fines de desarrollar habilidades y destrezas para aprender, aprender a pensar, aprender a hacer, aprender a ser, como pilares fundamentales de la educación y del proceso de aprendizajes que posibilitan a estudiantes a alcanzar el éxito en la sociedad actual. Puesto que, los docentes propician que los estudiantes aprendan de manera reflexiva, crítica y creativa, haciendo uso de estrategias, que propicie el trabajo en equipo, aprendizajes, saberes, cooperando desde sus capacidades; considerando las características individuales, socioculturales, lingüísticas; y las necesidades de sus estudiantes (Ministerio de Educación, 2014).

Por lo que, la institución formadora de docentes tiene la responsabilidad de desarrollar habilidades, capacidades en el uso de estrategias adecuadas y pertinentes para solucionar problemas, tomar 
decisiones, desarrollo de pensamiento reflexivo y pensamiento creativo, en los estudiantes para que de esta manera puedan responder a los procesos pedagógicos. Siendo estos procesos pedagógicos, "conjunto de prácticas, relaciones reciprocas y saberes que acontecen entre los participantes de los procesos educativos dentro de un ambiente de aprendizaje determinado con la finalidad de construir conocimientos, fortalecer valores, potenciar habilidades y destrezas" (Sánchez, 2021, p. 49). Encontrándose, como parte de este proceso el conflicto cognitivo, saberes previos, motivación, problematización, propósito, gestión y acompañamiento pedagógico para la construcción de los aprendizajes, metacognición y la retroalimentación. Para este caso se realiza hincapié en algunos de ellos por su preponderancia e importancia en el desarrollo de capacidades y competencias de aprendizajes.

Al respecto, es necesario tener en consideración la investigación de Gilar-Corbi (2003) "Adquisición de habilidades cognitivas: factores en el desarrollo inicial de la competencia experta", como parte de sus conclusiones hace mención: que las habilidades intelectuales tienen una influencia considerable en la adquisición de los conocimientos y habilidades que configuran la competencia experta, asimismo; que el aspecto motivacional implicado en la adquisición de la competencia es un tipo de motivación ligado a factores biológicos y temperamentales relacionados con el impulso general de la actividad, más que un tipo de motivación cognitiva, como un esfuerzo continuado por conseguir unas metas y con el objetivo de mejorar la ejecución.

De ahí que, el conflicto cognitivo es la inseguridad que nace en el estudiante al momento de enfrentarse a una situación problemática o desconocida que demanda otorgar respuestas, y al no poseer dentro de sus conocimientos previos la información que satisfaga, se ve en la necesidad de investigar y profundizar (Delgado, 2017), algo que "contribuyen significativamente al proceso de toma de decisiones mediante la creación de alternativas asertivas y funcionales vinculadas con la exploración de nuevas ideas o supuestos" (López et al., 2020, p. 406). Asimismo, en las acciones pedagógicas se debe de considerar que, para realizar el conflicto cognitivo se debe hacer dentro de una sesión de aprendizaje donde se plantea un problema al estudiante para generar el conflicto partiendo de preguntas que ayuden a identificar el contraste entre sus conocimientos previos y el nuevo conocimiento que va adquirir (Portal, 2017), para lograr que los estudiantes expuestos en clase sean más explicativos, y desarrollen las competencias transversales y específicas para que puedan enfrentarse a situaciones de la vida real (Cobos, 2019).

Por tanto, el conflicto cognitivo como proceso pedagógico en los estudiantes universitarios requiere la aplicación de estrategias metodológicas con actividades de aprendizaje donde se enfatice acciones para generar la metamotivación en el desarrollo de capacidades, habilidades y actitudes que permita reflexionar sobre el actuar de los educandos, frente a las situaciones de aprendizaje, asimismo; el conflicto cognitivo está presente en las actividades pedagógicas, psicológicas y sociales. En lo pedagógico; en los momentos de inicio, desarrollo y cierre de una sesión de aprendizaje, con la finalidad de buscar nuevos saberes para el desarrollo de conocimientos, capacidades 
y habilidades, que permita la modificación conceptual, a través del proceso de aprender, reaprender y desaprender, según las necesidades e intereses de aprendizaje, respecto a lo psicológico; en las actitudes y la motivación que van a determinar las metas y objetivos a lograr, donde el equilibro y desequilibrio mental generados por el conflicto cognitivo, permita la asimilación y acomodación cognitiva para la toma de decisiones pertinentes, $y$ en lo social; en respuestas a los valores y aspiraciones individuales o sociales según su contexto, a través de las relaciones humanas y la convivencia social que permita solucionar las necesidades de aprendizaje, según las metas personales o comunes.

Consecuentemente, lo que permite establecer las estrategias. Las situaciones problemáticas, donde la negociación, es un proceso para la resolución óptima de conflictos, que puede implementarse en las instituciones escolares cuando las personas o los grupos en situación problemática aspiran a recuperar o alcanzar una conveniencia relacional (González, 2017). Los nuevos saberes o "la construcción de nuevos conocimientos, parte de la necesidad de dar respuestas a situaciones concretas o problemas planteados, que necesitan la ampliación de las estructuras del conocimiento" (Aguilar y Oktac, 2004 citado en Delgado, 2017, p. 15). Las necesidades de aprendizajes "se refieren a las competencias o lo que las personas necesitan saber acerca del aprendizaje en sí para conseguir el éxito en lo que aprenden" (Gutiérrez, 2018, p. 94).

Por lo que, el conflicto cognitivo como estrategia permite realizar diversas actividades para la construcción de los aprendizajes, donde la confrontación o ruptura cognoscitiva va a admitir la aclaración de las dudas, dificultades para la toma de decisiones oportunas y pertinentes en el proceso de enseñanza y aprendizaje. De modo que, la función del docente como facilitador o guía, va a determinar las actividades y estrategias adecuadas para responder a las necesidades de aprendizaje de manera reflexiva.

La metamotivación es un proceso psicológico que permite moverse, poner en movimiento, listo para acción, y desde la óptica metacognitiva, es un estado interno compuesto por necesidades que genera interés, sistema de valores, corporación $\mathrm{y}$ actitudes que activa, dirige y mantiene la conducta humana (Cuba, 2019), a través de "las estrategias para aprender a aprender, como es la metacognición" (Yilmaz y Baydas, 2017, citado en Ros y Rodríguez, 2021, p. 465), de esta manera; se dirige a la satisfacción del deseo y aumenta la tensión, favoreciendo así el desarrollo de la persona. La motivación y metamotivación, son los móviles fundamentales que llevan al individuo al desarrollo de su personalidad y a escalar en la jerarquía de las necesidades (Elizalde et al., 2006), puesto que; la capacidad metamotivacional del sujeto, está asociado con la capacidad de producir y sostener un nivel de motivación consciente y, por lo mismo, susceptible de autorregulación (Tolentino, 2019).

Por lo tanto, la metamotivación es el estado interno de una persona bajo sus intereses, aspiraciones, actitudes personales y sociales, para ello; moviliza sus conocimientos, emociones internas y externas que lo motivan a tomar decisiones prudentes para enfrentarse a situaciones problemáticas y resolverlo, de esta manera permitiendo su crecimiento y desarrollo del estudiante. 
Considerando el enfoque de las capacidades, las personas son sujetos con posibilidades, dueños de sus acciones y del rumbo de sus vidas, mejorando las condiciones de acceso y dándole un lugar privilegiado a los derechos y a las libertades individuales (Silva \& Mazuera, 2019), de ahí que; el desarrollo de capacidades es un "proceso de desarrollo individual que conduce a niveles más altos de habilidad" (Moore, Crozier \& Kite, 2012; Trostle, 1992, citado en Pástor et al., 2020, p. 7), "mediante el cual las personas, organizaciones y sociedades obtienen, fortalecen y mantienen las aptitudes necesarias para establecer y alcanzar sus propios objetivos de desarrollo a lo largo del tiempo" (Programa de las Naciones Unidas para el Desarrollo, 2009, p. 3). Además, el desarrollo de capacidades "consiste en mejorar las condiciones, conseguir un mejor desempeño, agregar valor añadido, desarrollar nuevos talentos en las que se desenvuelven las personas para que puedan exprimir todo su potencial" (Rueda, 2014, p. 6), por lo que; es esencial en el trabajo de acompañamiento a las personas y organizaciones que viven con el desasosiego de promover cambios sostenibles e incluyentes en sus respectivas sociedades, y que necesitan fortalecer sus capacidades para un desempeño más eficaz (ALBOAN, 2015). De modo que, la adquisición del conocimiento, como el manejo de habilidades van a depender, de los saberes previos y su relación en la adquisición con los nuevos conocimientos (Acero y Loyola, 2019).

Por consiguiente, el desarrollo de capacidades es lograr ampliamente y de manera divergente los conocimientos, habilidades y actitudes para dar respuesta, atender y solucionar diversas situaciones, por lo que; la metamotivación será determinante para lograr estas capacidades como una actividad satisfactoria, logrando reflexionar sobre las situaciones de aprendizaje.

La importancia de la investigación, radica por su fundamento teórico y práctica en: el aspecto pedagógico, su implicancia en el proceso de enseñanza y aprendizaje, en sus procesos pedagógicos porque va a permitir fundamentar sobre el conflicto cognitivo y su relación con la metamotivación, para el desarrollo de capacidades en los estudiantes; en el aspecto psicológico, en lo actitudinal y motivacional, determinante para los aprendizajes; en el aspecto individual y social con respecto a las aspiraciones personales o comunes para el desarrollo de las capacidades y logro de los aprendizajes.

El objetivo principal del estudio, fue relacionar el conflicto cognitivo con la metamotivación para determinar el impacto de esta con el desarrollo de capacidades en los estudiantes universitario, para ello se tuvo que comprobar la relación de las situaciones problemáticas, los nuevos saberes y las necesidades de aprendizajes, como estrategias del conflicto cognitivo, en la metamotivación para el desarrollo de capacidades.

\section{MÉTODO}

La investigación según el propósito fue de tipo aplicado, el nivel de investigación para este caso fue explicativo y causal, identifica la causa y efecto o resultado, así como factores, variables o conceptos intervinientes o moderadores (Hernández-Sampieri y Mendoza, 2018), asimismo; el diseño permitió manipular la variable independiente en relación con la dependiente y su implicancia, para este estudio fue importante trabajar la diversidad de estrategias de conflicto cognitivo en la metamotivación para el desarrollo de capacidades. 
Se utilizó el método científico, "método de conocimiento que integra la inducción y la deducción, con la finalidad de construir conocimiento teórico y aplicado" (Sánchez et al. 2018, p. 89), lo que permitió los procedimientos a lo largo de la investigación, además; se utilizó el método específico hipotético deductivo para la obtención de información determinada a partir del planteamiento de los objetivos, que fue trabajado en estricta relación con los resultados obtenidos, según los grupos de la muestra materia de investigación.

La población estuvo conformada por 95 estudiantes de la Facultad de Ciencias de la Educación de la Universidad Nacional Daniel Alcides Carrión, Perú. Luego se precisó la muestra de tipo directa intencional con grupos completos, 18 estudiantes del grupo experimental y 14 estudiantes del grupo de control que representa $30 \%$ de los estudiantes de la población determinada.

Se elaboró un cuestionario compuesto por 12 preguntas, después se procedió a la confiabilidad al 95\% a través de Alfa de Cronbach "se pudo estimar la fiabilidad de la consistencia interna del instrumento" (Hernández y Pascual, 2018, p. 159), obteniendo el coeficiente de 0.82 de confiabilidad alta, además; se realizó la prueba de validación y la de piloto, logrando resultado favorable. Luego se aplicó dicho cuestionario a través de un formulario virtual; para determinar la relación del conflicto cognitivo con la metamotivación. Seguidamente, se empleó una prueba pedagógica compuesta por 20 interrogantes, antes y después, dirigido al grupo experimental y de control, para determinar el impacto de la metamotivación en el desarrollo de capacidades, para lo cual; se usó el programa Office Excel 2016, el cual nos permitió ordenar los datos y el SPSS versión 25 que generó las tablas y los cálculos respectivos.

\section{RESULTADOS}

Se realizó la correlación del conflicto cognitivo $\mathrm{y}$ sus respectivas dimensiones (situaciones problemáticas, nuevos saberes y necesidades de aprendizajes) con la metamotivación, para garantizar la significancia entre ambas variables, que permitió posteriormente determinar el impacto de la metamotivación en el desarrollo de capacidades en el grupo experimental y de control, tanto en el pretest y postest, al respecto se procedió con la homogeneización con los datos de la varianza y la validación según el objetivo de estudio.

Tabla 1. Las situaciones problemáticas en la metamotivación.

\begin{tabular}{|c|c|c|c|c|}
\hline & & & Metamotivación & $\begin{array}{c}\text { Situaciones } \\
\text { problemáticas }\end{array}$ \\
\hline \multirow[t]{6}{*}{ Rho de Spearman } & Metamotivación & Coeficiente de correlación & 1,000 &, $646^{\star *}$ \\
\hline & & Sig. (bilateral) & . &, 000 \\
\hline & & $\mathrm{N}$ & 32 & 32 \\
\hline & Situaciones problemáticas & Coeficiente de correlación &, $646^{\star \star}$ & 1,000 \\
\hline & & Sig. (bilateral) &, 000 & . \\
\hline & & $\mathrm{N}$ & 32 & 32 \\
\hline
\end{tabular}

\footnotetext{
**. La correlación es significativa en el nivel 0,01 (bilateral).
} 
Los datos de la Tabla 1 precedente, muestran que las situaciones problemáticas en la metamotivación, es de 0,646 según la correlacional Rho de Spearman; el p valor es 0,000 0,05 indicando una relación moderada y significativa.

Tabla 2. Los nuevos saberes en la metamotivación.

\begin{tabular}{|c|c|c|c|c|}
\hline & & & Metamotivación & Nuevos saberes \\
\hline \multirow[t]{6}{*}{ Rho de Spearman } & Metamotivación & Coeficiente de correlación & 1,000 &, $578^{\star \star}$ \\
\hline & & Sig. (bilateral) & . & 001 \\
\hline & & $\mathrm{N}$ & 32 & 32 \\
\hline & Nuevos saberes & Coeficiente de correlación &, $578^{\star \star}$ & 1,000 \\
\hline & & Sig. (bilateral) & 001 & . \\
\hline & & $\mathrm{N}$ & 32 & 32 \\
\hline
\end{tabular}

**. La correlación es significativa en el nivel 0,01 (bilateral).

Según los datos que antecede en la Tabla 2 muestran que los nuevos saberes en la metamotivación, es de 0,578 de acuerdo a la correlacional Rho de Spearman; p valor es 0,001<0,05 que indica una relación moderada y significativa.

Tabla 3. Las necesidades de aprendizaje en la metamotivación.

\begin{tabular}{|c|c|c|c|c|}
\hline & & & Metamotivación & $\begin{array}{l}\text { Necesidades } \\
\text { aprendizaje }\end{array}$ \\
\hline \multirow[t]{6}{*}{ Rho de Spearman } & Metamotivación & Coeficiente de correlación & 1,000 & ,304 \\
\hline & & Sig. (bilateral) & . & ,091 \\
\hline & & $\mathrm{N}$ & 32 & 32 \\
\hline & Necesidades aprendizaje & Coeficiente de correlación & ,304 & 1,000 \\
\hline & & Sig. (bilateral) & ,091 & . \\
\hline & & $\mathrm{N}$ & 32 & 32 \\
\hline
\end{tabular}

**. La correlación es significativa en el nivel 0,01 (bilateral).

Los datos de la Tabla 3 que antecede, muestran que las necesidades de aprendizaje en la metamotivación, es 0,304 con la correlacional Rho de Spearman; $p$ valor es 0,091>0,05 que indica una relación moderada no significativa. 
Tabla 4. Conflicto cognitivo en la metamotivación.

\begin{tabular}{lllcc}
\hline & & $\begin{array}{c}\text { Conflicto } \\
\text { cognitivo }\end{array}$ & Metamotivación \\
\hline Rho de Spearman & Metamotivación & Coeficiente de correlación & 1,000 &, $759^{* *}$ \\
& & Sig. (bilateral) & $\cdot$ &, 000 \\
& Metamotivación & Coeficiente de correlación & 32 & 32 \\
& & Sig. (bilateral) &, $759^{* *}$ & 1,000 \\
& $\mathrm{~N}$ &, 000 &. \\
& & & 32 & 32 \\
\hline
\end{tabular}

**. La correlación es significativa en el nivel 0,01 (bilateral).

Observando la Tabla 4, la correlación de Rho de Spearman es de 0,759, el p valor es $0,000<0,05$ que indica una alta correlación y significativa entre las variables en estudio, demostrando que a mayor uso de las estrategias del conflicto cognitivo es mayor metamotivación en los estudiantes universitarios.

Tabla 5. La metamotivación en el desarrollo de capacidades.

\begin{tabular}{|c|c|c|c|c|}
\hline & Grupo & $\mathbf{N}$ & Media & Desviación estándar \\
\hline \multirow[t]{2}{*}{ Pretest } & grupo control & 14 & 13,64 & 2,735 \\
\hline & grupo experimental & 18 & 15,94 & 1,349 \\
\hline \multirow[t]{2}{*}{ Pretest } & grupo control & 14 & 13,29 & 3,474 \\
\hline & grupo experimental & 18 & 16,94 & 1,211 \\
\hline
\end{tabular}

En la Tabla 5 precedente se observa que la media del grupo control en el pretest es de 13,64 y en el postest es de 13,29 existiendo una diferencia de $-0,35$; lo que significa que los rendimientos en lo referido han descendido, mientras; en el grupo experimental en el pretest 15,94 y en el postest de 16,94 ascendiendo 1,00. Además, la desviación estándar en el grupo de control en el pretest es de 2,735 y en postest 3,474 , lo que indica que los rendimientos son más heterogéneos, mientras; en el grupo experimental en el pretest es de 1,349 y en el postest a 1,211 , lo que indica que los resultados de aprendizajes sean más homogéneos, por acercamiento a la unidad; permitiendo el desarrollo de capacidades. 
Tabla 6. Homogeneidad de datos.

\begin{tabular}{llccccc}
\hline & & \multicolumn{2}{c}{$\begin{array}{c}\text { Prueba de Levene de } \\
\text { igualdad de varianzas }\end{array}$} & \multicolumn{2}{c}{ prueba t para la igualdad de medias } \\
& & F & Sig. & T & gl & Diferencia de medias \\
\hline Pretest & Se asumen varianzas iguales & 16,049 &, 000 & $-3,125$ & 30 & $-2,3$ \\
& No se asumen varianzas iguales & & & $-2,888$ & 17,898 & $-2,3$ \\
\multirow{2}{*}{ Postest } & Se asumen varianzas iguales & 28,605 &, 000 & $-4,171$ & 30 & $-3,65$ \\
& No se asumen varianzas iguales & & & $-3,767$ & 15,470 & $-3,65$ \\
\hline
\end{tabular}

\section{Procedimientos para su determinación}

1. Criterios para la prueba de homogeneidad:

$$
\begin{aligned}
& \mathrm{H}_{0}: \sigma_{1}^{2}=\sigma_{2}^{2} \\
& \mathrm{H}_{\mathrm{a}}: \sigma_{1}^{2} \neq \sigma_{2}^{2}
\end{aligned}
$$

\section{Nivel de significancia:}

$\alpha=0,05(5 \%)$

\section{Prueba estadística:}

Prueba de Levene

Analizar / comparar medias / Prueba t para muestras independientes / paso pretest / defino grupos

\section{Criterio de decisión}

Si $p<0,05$ rechazamos la $h_{0}$ y aceptamos la $h_{a}$ Si p $>0,05$ rechazamos la $h_{a}$ y aceptamos la $h_{0}$

- Valor de $\mathrm{p}=0,000$

Comparación de p y a

- $\mathrm{p}$ valor $=0,000<\alpha=0,05$

\section{Resultados y conclusión:}

Como $\mathrm{p}=0,000<0,05$ entonces se acepta la $\mathrm{H}_{0}$ y se rechaza la $\mathrm{H}_{\mathrm{a}}$, es decir las varianzas de los grupos son homogéneos, demostrando la validez del propósito de estudio.

Del mismo modo, se observa en la Tabla 6 precedente que el valor de la prueba $t$ es $-3,125$ en el pretest y $-4,171$ en el postest con 30 grados de libertad; indicando el valor según la tabla $t$ de Student de 2,042, por lo que; de acuerdo a los resultados obtenidos se ubica fuera del rango de validez de la hipótesis nula, validando la hipótesis de investigación, de manera que; el conflicto cognitivo tiene impacto positivo en la metamotivación determinando el desarrollo de capacidades profesionales, en los alumnos universitarios, tal como se muestra en el Gráfico 1. 


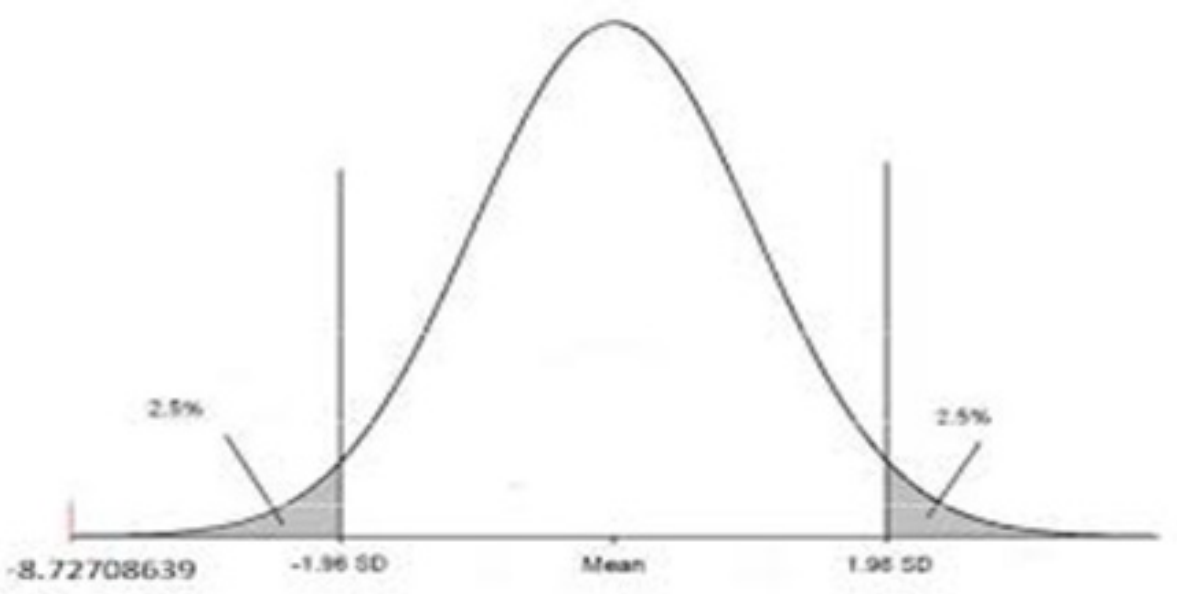

Gráfico 1. Campana de Gauss con dos colas.

\section{Discusión}

Con respecto a la correlación según Rho de Spearman fue 0,759 que indica alta correlación entre las variables en estudio, demostrando que a mayor aplicación del conflicto cognitivo hay mayor metamotivación en los estudiantes universitarios, además se observó que la significación bilateral es 0,000 lo que indica un valor significativo.

Según los datos (Tabla 5), los resultados de la media en el grupo de control tienden a bajar, mientras en el grupo experimental tiende a subir, por lo que; a menor uso de estrategias de conflicto cognitivo poca motivación y resultados poco favorables, mientras a mayor uso de estrategias de conflicto cognitivo mayor motivación logrando resultados favorables y significativos, además, los resultados son más homogéneos como se evidencia en la desviación estándar de la respectiva tabla.

Según la investigación de Gilar-Corbi (2003), hace referencia que el aspecto motivacional implica en la adquisición de la competencia y relacionados con el impulso general de la actividad por conseguir unas metas, con el objetivo de mejorar la ejecución, lo cual; corrobora con los resultados de la presente investigación donde la motivación y la metamotivación permite e impacta en desarrollar capacidades y mejora el rendimiento en los aprendizajes de los estudiantes de la Facultad de Ciencias de la Educación de la Universidad Nacional Daniel Alcides Carrión. De ahí que, a mayor uso de estrategias relacionadas con el conflicto cognitivo tiene impacto positivo en la metamotivación determinando el desarrollo de capacidades profesionales en los alumnos universitarios.

\section{CONCLUSIONES}

Las situaciones problemáticas, los nuevos saberes, las necesidades de aprendizaje, como estrategias influyen en la metamotivación, lo que permite el desarrollo de capacidades y la obtención de mejores resultados de aprendizaje.

El conflicto cognitivo se encuentra relacionado con la metamotivación, según la correlación Rho de Spearman es de 0,759 lo que significa alta relación y significativa, por lo que; la construcción de los aprendizajes va a depender de las estrategias 
pertinentes, teniendo en cuenta el aspecto pedagógico, psicológico y social.

La metamotivación tiene un impacto significativo en el desarrollo de capacidades, logrando resultados más homogéneos, cuyo valor 0,000 indica que al 95\% son definitivamente válidos, lo que debe ser priorizado para la construcción de los aprendizajes y obtener rendimientos académicos favorables en los estudiantes.

La relación entre las estrategias del conflicto cognitivo y la metamotivación permite el desarrollo y fortalecimiento de capacidades, en la medida que estas sean usadas en los procesos pedagógicos, en plena combinación con los conocimientos, habilidades, actitudes.

\section{REFERENCIAS}

Acero, A. M., y Loyola, G. (2019). Los saberes previos y la comprensión lectora en los estudiantes del sexto ciclo del Programa Beca 18 de la Universidad Católica Sedes Sapientiae - Lima, 2017. [Tesis de maestría, Universidad Católica Sedes Sapientiae]. http://repositorio.ucss.edu. pe/handle/UCSS/744

ALBOAN (2015). El desarrollo de capacidades como estrategia de cambio. Una experiencia colectiva de búsqueda de alternativas. ALBOAN. https:// dhls.hegoa.ehu.eus/documents/5511

Cobos, M. (2019). Empleo del conflicto cognitivo y metodología expositiva para la unidad didáctica de materiales poliméricos en $2^{\circ}$ de Bachillerato. [Trabajo de máster, Universidad Internacional de la Rioja]. https://reunir.unir. net/handle/123456789/8771

Cuba, P. k. (2019). Estrategias psicodidácticas para mejorar habilidades metacognitivas en los estudiantes del 5to "A" de secundaria en el área de comunicación de la I.E. "Inmaculada Concepción" del distrito de J.L.B. y Rivero, Arequipa 2018. [Tesis para optar título, Universidad Nacional San Agustín]. http://repositorio.unsa.edu.pe/ handle/UNSA/8573
Delgado, D. I. (2017). El trabajo cooperativo como una estrategia en la generación de conflictos cognitivos. [Trabajo de titulación, Universidad de Cuenca]. http://dspace.ucuenca.edu.ec/ handle/123456789/28171

Elizalde, A., Martí, M., y Martínez, F. A. (2006). Una revisión crítica del debate sobre las necesidades humanas desde el Enfoque Centrado en la Persona. Polis, Revista de la Universidad Bolivariana, 5(15). https://www.redalyc.org/ pdf/305/30517306006.pdf

González, R. A. (2017). Estrategias para abordar los conflictos en el aula de clase. [Tesis de maestría, Universidad de Antioquia]. https://bibliotecadigital.udea.edu.co/ handle/10495/9536

Gutiérrez, M. (2018). Estilos de aprendizaje, estrategias para enseñar. Su relación con el desarrollo emocional y "aprender a aprender”. Tendencias Pedagógicas, 31, 83-96. https://doi.org/10.15366/tp2018.31.004

Hernández-Sampieri, R., y Mendoza, C. P. (2018). Metodología de la investigación: las rutas cuantitativa, cualitativa y mixta. McGraw-Hill Interamericana Editores, S.A. de C. V

Hernández, H. A. y Pascual, A. E. (2018). Validación de un instrumento de investigación para el diseño de una metodología de autoevaluación del sistema de gestión ambiental. Revista de Investigación Agraria y Ambiental, 9(1), 157163. https://doi.org/10.22490/21456453.2186

Ley No 28044 (2003). Ley general de educación. Plataforma digital única del Estado Peruano. https://n9.cl/rl190

López, D., Guamán, M., y Castro, J. (2020). La toma de decisiones y la eficacia organizativa en las Pymes comerciales de la ciudad de Ambato (Ecuador). Revista Espacios 41(22), 396-409. https://n9.cl/3jkuu7

Ministerio de Educación (2014). Marco del buen desempeño docente. Corporación Gráfica Navarrete.

Ministerio de educación (2005). Diseño curricular nacional de educación básica regular - proceso de articulación. Ministerio de Educación del Perú. 
Portal, K. J. (2017). El Conflicto cognitivo como reto. Universidad Peruana Cayetano Heredia. https:// n9.cl/187s7

Pástor, D., Arcos, G. de L., y Domínguez, A. L. (2020). Desarrollo de capacidades de investigación para estudiantes universitarios mediante el uso de estrategias instruccionales en entornos virtuales de aprendizaje. Apertura, 12(1), 6-21. http:// dx.doi.org/10.32870/Ap.v12n1.1842

Programa de las Naciones Unidas para el Desarrollo (2009). Desarrollo de capacidades (texto básico del PNUD). PNUD. https://n9.cl/7dyas

Ros, G., y Rodríguez, M. T. (2021). Influencia del aula invertida en la formación científica inicial de Maestros/as: beneficios en el proceso de enseñanza - aprendizaje, actitudes y expectativas hacia las ciencias. Revista de Investigación Educativa, 39(2), 463-482. http://dx.doi. org/10.6018/rie.434131

Rueda, J. (2014). El desarrollo de capacidades un enfoque central de la cooperación al desarrollo. Universitat de Barcelona. http://hdl.handle. net $/ 2445 / 66912$

Sánchez, G. H. (2021). Procesos pedagógicos y curriculares de la red de educadores "cómplices pedagógicos latinoamericanos" en tiempos pandemia. [Tesis de maestría, Universidad de la Costa]. https://hdl.handle.net/11323/8478

Sánchez, H., Reyes, C. y Mejía, K. (2018). Manual de términos en investigación científica, tecnológica y humanística. Universidad Ricardo Palma

Silva, W. H. y Mazuera, J. A. (2019). ¿Enfoque de competencias o enfoque de capacidades en la escuela? Revista Electrónica de Investigación Educativa, 21(e17), 1-10. https://doi. org/10.24320/redie.2019.21.e17.1981
Organización de las Naciones Unidas para la Educación, la Ciencia y la Cultura (2005). Hacia las sociedades del conocimiento: informe mundial de la UNESCO. Ediciones UNESCO. https:// unesdoc.unesco.org/ark:/48223/pf0000141908

Gilar-Corbi, R. (2003). Adquisición de habilidades cognitivas: factores en el desarrollo inicial de la competencia experta. [Tesis de doctorado. Universidad de Alicante]. http://hdl.handle. net/10045/9906

Tolentino, L. A. (2019). Factores personales $e$ institucionales asociados a la dificultad para elaborar la tesis en el Programa de Doctorado de la Facultad de Educación de la Universidad Nacional Mayor de San Marcos - 2017. [Tesis de doctorado, Universidad Nacional Mayor de San Marcos]. https://hdl.handle. net/20.500.12672/11255

Conflictos de intereses. Los autores declaran que no existen conflictos de intereses.

Contribución autoral. Teófilo Félix Valentín Melgarejo. realizó la sistematización, redactó el artículo y corrigió el estilo según norma de redacción. Oscar Cirilo Rivera Trujillo. Realizó la búsqueda de información sobre conflicto cognitivo y la metamotivación.

José Rovino Alvarez López. Realizó el procesamiento de los datos y la sistematización de los resultados. Rosa Luz Gómez Segura. Realizó el trabajo metodológico, apoyo en el procesamiento y sistematización de los datos. Gastón Jeremías Oscátegui Nájera. Realizó la búsqueda información sobre desarrollo de capacidades.

Además, todos los autores trabajaron de manera activa y consensuada el artículo. Por lo que; aprobamos para su evaluados y publicación. 